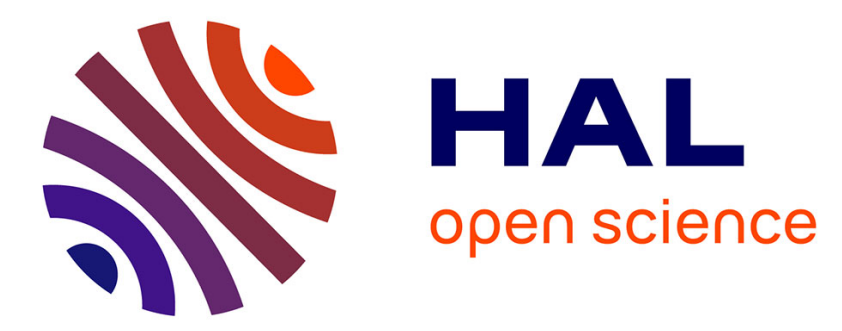

\title{
Complex convective structures in three- dimensional binary fluid convection in a porous medium
}

\author{
David Lo Jacono, Alain Bergeon, Edgar Knobloch
}

\section{To cite this version:}

David Lo Jacono, Alain Bergeon, Edgar Knobloch. Complex convective structures in three- dimensional binary fluid convection in a porous medium. Fluid Dynamics Research, 2017, 49 (6), pp.061402. 10.1088/1873-7005/aa7b2a . hal-01729108

\section{HAL Id: hal-01729108 \\ https://hal.science/hal-01729108}

Submitted on 12 Mar 2018

HAL is a multi-disciplinary open access archive for the deposit and dissemination of scientific research documents, whether they are published or not. The documents may come from teaching and research institutions in France or abroad, or from public or private research centers.
L'archive ouverte pluridisciplinaire HAL, est destinée au dépôt et à la diffusion de documents scientifiques de niveau recherche, publiés ou non, émanant des établissements d'enseignement et de recherche français ou étrangers, des laboratoires publics ou privés. 


\section{Open Archive TOULOUSE Archive Ouverte (OATAO)}

OATAO is an open access repository that collects the work of Toulouse researchers and makes it freely available over the web where possible.

This is an author-deposited version published in : http://oatao.univ-toulouse.fr/ Eprints ID : 19647

To link to this article : DOI:10.1088/1873-7005/aa7b2a

URL : https://doi.org/10.1088/1873-7005/aa7b2a

To cite this version : Lo Jacono, David Knobloch, Edgar Complex convective structures in three- dimensional binary fluid convection in a porous medium. (2017) Fluid Dynamics Research, vol. 49. pp. 061402/1-061402/22. ISSN 0169-5983

Any correspondence concerning this service should be sent to the repository administrator: staff-oatao@listes-diff.inp-toulouse.fr 


\title{
Complex convective structures in three- dimensional binary fluid convection in a porous medium
}

\author{
D Lo Jacono ${ }^{1}$, A Bergeon ${ }^{1}$ and E Knobloch ${ }^{2}$ \\ ${ }^{1}$ Institut de Mécanique des Fluides de Toulouse (IMFT), Université de Toulouse, \\ CNRS, INPT, UPS, Toulouse, France \\ ${ }^{2}$ Department of Physics, University of California, Berkeley CA 94720, United States \\ of America
}

\begin{abstract}
Three-dimensional convection in a binary mixture in a porous medium heated from below is studied. For negative separation ratios steady spatially localized convection patterns are expected. Such patterns, localized in two dimensions, are computed in square domains with periodic boundary conditions and three different aspect ratios. Numerical continuation is used to examine their growth as the Rayleigh number varies and to study their interaction with their images under periodic replication once they reach the size of the domain. In relatively small domains with six critical wavelengths on a side three different structures are found, two with four arms extended either along the principal axes or the diagonals and one with eight arms. As these states are followed as a function of the Rayleigh number all succeed in growing so as to ultimately fill the domain thereby generating an extended convection pattern. In contrast, in larger domains, with 12 or 18 critical wavelengths on a side, the solution branches undergo much more complex behavior but appear to fail to generate spatially extended states. In each case both $D_{4^{-}}$and $D_{2}$-symmetric structures are computed.
\end{abstract}

Keywords: spatially localized structures, convection, bifurcation 


\section{Introduction}

Stationary spatially localized states are of great interest in the theory of pattern formation. Recently two-dimensional states of this type, localized in one dimension, have been found in several different types of convection, including binary fluid convection (Batiste and Knobloch 2005, Batiste et al 2006), convection in an imposed magnetic field (Blanchflower 1999, Dawes 2007) and natural doubly diffusive convection (Ghorayeb 1997, Ghorayeb and Mojtabi 1997, Bergeon and Knobloch 2008a, 2008b, Bergeon et al 2008). Similar states, localized in the cross-stream direction, have been identified in plane Couette flow (Schneider et al 2010a). In these systems, localized states occur inside the region of coexistence between a spatially homogeneous state and a spatially periodic state, in a parameter interval called the snaking or pinning region (Burke and Knobloch 2007, Knobloch 2015). This region also contains multiple states resembling bound states of single pulse localized states, as demonstrated recently in the context of binary fluid convection with a negative separation ratio in a porous medium (Lo Jacono et al 2010). The $1+1$-dimensional Swift-Hohenberg equation provides a convenient model equation that sheds much light on the origin and properties of the pinning region in these systems (Burke and Knobloch 2007).

It is natural to consider the existence of three-dimensional structures that are localized in two spatial dimensions. Structures of this type were originally found in magnetoconvection (Blanchflower and Weiss 2002) but attempts to find stable states of this type in binary fluid convection failed although meandering structures with slow but complex time-dependence were identified in direct numerical simulations of the governing equations (Mercader et al 2008, Alonso et al 2014). Related but highly anisotropic structures resembling turbulent puffs were recently computed for plane Couette flow (Duguet et al 2009, Schneider et al 2010b). In contrast, the Swift-Hohenberg equation, hereafter SH23 or SH35 depending on the nonlinear terms (Burke and Knobloch 2007), is isotropic and has variational structure in any number of dimensions. Its solutions consequently evolve to steady states corresponding to local minima of the free energy. In $2+1$ dimensions these minima may correspond to structures that are fully localized in two dimensions (Lloyd et al 2008, Avitabile et al 2010). These structures can take the form of spatially localized patches of hexagons or spatially localized patches of rolls, dubbed 'worms' owing to their segmented appearance, depending on the symmetry properties of the nonlinear terms (Lloyd et al 2008, Avitabile et al 2010). Localized target patterns can also be found as can localized spots. Of these the hexagons, worms and targets are all found in the subcritical regime where a periodic pattern, be it hexagons or rolls, coexists with the trivial solution. As in one dimension, all these patterns 'snake', at least initially, as the structure is followed in parameter space and grows by nucleating additional cells or rings along its periphery (Lloyd et al 2008, Lloyd and Sandstede 2009). Spot-like states differ in that they are present even in the supercritical regime (Lloyd and Sandstede 2009), and related states have been observed in nonvariational systems of equations, such as reaction-diffusion equations (Coullet et al 2000) or the equations arising in nonlinear optics (McSloy et al 2002).

In this paper we are interested in localized states with exotic structure in binary fluid convection in a porous medium. In earlier work (Lo Jacono et al 2013) we have computed, using numerical continuation, steady localized states in the form of a cross and argued that states of this type exist and can be stable because of the confining concentration field whose gradients have been expelled from regions of active convection. In the present work we build on these earlier results in several ways. In the first instance we study periodic domains of several aspect ratios, starting with a $6 \lambda_{c} \times 6 \lambda_{c} \times 1$ domain, and then presenting detailed results for a $12 \lambda_{c} \times 12 \lambda_{c} \times 1$ domain. These results are compared with those obtained in an $18 \lambda_{c} \times 18 \lambda_{c} \times 1$ domain reported in Lo Jacono et al (2013). Here $\lambda_{c}$ is the onset wavelength 
predicted by linear theory. The use of smaller domains in this work allows us to study the behavior of the localized states when they start to interact with their images and so cease to be isolated localized structures. Such a study is of considerable interest since it sheds light on the manner in which the localized states grow in spatial extent in an attempt to generate a domainfilling convecting state. Our study reveals a number of surprises of which convecting states with a 'starfish' structure are perhaps the most remarkable.

Throughout we use the term localized to refer to structures that have no or weak interaction with their images under periodic replication; in practice, this means that their amplitude within one wavelength of the domain boundary is small.

\section{Statement of the problem}

Following Lo Jacono et al (2013) we study binary convection with a negative separation ratio in a porous medium. The dimensionless equations describing this system are

$$
\begin{aligned}
& 0=-\nabla p+R a(T+S C) \mathbf{e}_{z}-\mathbf{u}, \\
& 0=\nabla \cdot \mathbf{u}, \\
& \frac{\partial T}{\partial t}=-(\mathbf{u} \cdot \nabla) T+\nabla^{2} T, \\
& \epsilon \frac{\partial C}{\partial t}=-(\mathbf{u} \cdot \nabla) C+\tau \nabla^{2}(C-T),
\end{aligned}
$$

where $\mathbf{u}=(u, v, w)$ and $\nabla \equiv\left(\partial_{x}, \partial_{y}, \partial_{z}\right)$ in $(x, y, z)$ coordinates, with $(x, y)$ in the horizontal direction and $z$ in the vertical; $T$ is the temperature and $C$ is the concentration of the heavier component of the mixture. The (inverse) Lewis number $\tau$, the Rayleigh number $R a$ and the separation ratio $S$ are defined by

$$
\tau=\frac{D}{\kappa}, \quad R a=\frac{g\left|\rho_{T}\right| \Delta T h}{\lambda \kappa}, \quad S=-S_{\text {Soret }} C_{0}\left(1-C_{0}\right) \frac{\rho_{\mathrm{C}}}{\rho_{\mathrm{T}}}<0,
$$

where $\lambda$ is the Darcy friction coefficient, $\kappa$ is the thermal diffusivity, $D$ is the solute diffusivity, $S_{\text {Soret }}<0$ is the Soret coefficient, $g$ is the gravitational acceleration and $h$ is the depth of the layer. The parameter $\epsilon$ denotes the porosity. In writing these equations we have used the layer depth $h$ as a unit of length and the vertical thermal diffusion time $h^{2} / \kappa$ as the unit of time, together with the linearized equation of state, $\rho(T, C)=\rho_{0}+\rho_{\mathrm{T}}\left(T-T_{0}\right)$ $+\rho_{\mathrm{C}}\left(C-C_{0}\right)$, where $\rho_{\mathrm{T}}<0, \rho_{\mathrm{C}}>0$ and the subscript zero indicates reference values. We suppose that a temperature difference $\Delta T>0$ is imposed across the system with the lower boundary hotter than the upper boundary; in response the system develops a concentration difference $\Delta C=\left|S_{\text {Soret }}\right| C_{0}\left(1-C_{0}\right) \Delta T$ with $C$ larger at the bottom than at the top. We use $\Delta T$ and $\Delta C$ as units of temperature and concentration.

Since the mass flux is proportional to the gradient of $C-T$ the boundary conditions read at $z=0: \quad w=T-1=(C-T)_{z}=0, \quad$ at $z=1: \quad w=T=(C-T)_{z}=0$,

together with periodic boundary conditions with dimensionless period $\Gamma$ in the $(x, y)$ directions. Note that because of the Darcy friction law we cannot impose boundary conditions on $(u, v)$ at $z=0,1$. Thus the velocity boundary conditions are 'stress-free'. This property of the system is beneficial for numerical continuation; the absence of nonlinear terms in the equation of motion (the effective Prandtl number in the medium is infinite) also helps. 
In the following we write $T=1-z+\Theta, C=1-z+\Sigma$ and examine the properties of the equations for $u, v, w, \Theta$ and $\Sigma$ all of which vanish in the conduction state. These equations are equivariant under translations in $(x, y)$ modulo the period $\Gamma$. In addition, they are equivariant under the reflection $(x, y) \rightarrow(-x, y)$ and the $90^{\circ}$ rotation $(x, y) \rightarrow(-y, x)$. Together these symmetries generate the symmetry group $D_{4} \dot{+} T^{2}$, where $D_{4}$ is the symmetry of a square, $T^{2}$ denotes a two-torus of translations and the symbol $\dot{+}$ indicates the semidirect product (Silber and Knobloch 1988). Finally, with the boundary conditions (6) the equations are also equivariant with respect to the midplane reflection $z \rightarrow 1-z$. The latter symmetry favors roll-like patterns (Golubitsky et al 1984, Umla et al 2010) and therefore plays an essential role in the observed patterns.

We solve equations (1)-(4) with the boundary conditions (6) on a square domain with period $\Gamma$ in two orthogonal directions in the horizontal for the representative parameter values $\epsilon=1, \tau=0.5, S=-0.1$ used in Lo Jacono et al (2013). For these parameter values the wavelength $\lambda_{c}$ of the primary mode is given by $\lambda_{c}=2 \pi / k_{c}$, where $k_{c} \approx 3.75$ is the onset wavenumber in an infinite system (Lo Jacono et al 2010), and the resulting bifurcation to rolls is subcritical. We use numerical continuation to follow steady states starting with a small amplitude (unstable) target pattern obtained by direct numerical simulation such as those shown in figures 5(b) and 8(a). At these small amplitudes the equations have a large number of distinct solutions with different symmetries that are, in large domains, exponentially close. The spectral element method used in our continuation favors solutions with $D_{4}$ symmetry (Assemat et al 2007), i.e., states that are invariant under reflection in the principal axes ( $x$ or $y$ axes) as well as reflection in the diagonals. Our continuation procedure is also capable of finding solutions with $D_{2}$ symmetry, i.e., solutions that are invariant under reflection in the $x$ or $y$ axes only (Lo Jacono et al 2013).

The continuation method used is that described in Lo Jacono et al (2013) and employs threedimensional spectral element spatial discretization that takes advantage of full tensorization of the Helmholtz and Poisson operators in the three spatial directions and the symmetry of the system to solve the problem in a quarter domain. The full domain solution is then generated by applying appropriate symmetry operations to this solution. Similar numerical implementation can be found in Beaume et al (2013a, 2013b). The quarter domain is decomposed into $N_{e} \times N_{e}$ spectral elements and in each element all fields are approximated by a high order interpolant through the Gauss-Lobatto-Legendre points. Resolution in the quarter domain varies from $N_{e}=6$ for $\Gamma=6 \lambda_{c}$ to $N_{e}=18$ for $\Gamma=18 \lambda_{c}$ with spatial resolution per element varying from $23 \times 23 \times 17$ points in each direction to $9 \times 9 \times 13$ points in each direction, respectively.

\section{Results: $6 \lambda_{c} \times 6 \lambda_{c} \times 1$ domain}

We begin by discussing first the results obtained in a relatively small domain. Three different types of states have been identified: states with the symmetry of the domain (i.e., $D_{4}$-symmetric states that are invariant under rotations by $90^{\circ}$ together with reflections in the principal axes and the diagonals) as well as states with (approximate) $D_{8}$ symmetry (i.e., states invariant under rotations by $45^{\circ}$ together with reflections in the principal axes and the diagonals) and $D_{2}$-symmetric states (i.e., states invariant under rotations by $180^{\circ}$ together with reflections in the principal axes). In each case it is necessary to distinguish between three types of states: (i) spatially localized states that make no contact with the domain boundary or have very small amplitude at the location of the boundary, (ii) spatially extended states with the same symmetry that interact with the image structures across the boundary but are not domain-filling, and (iii) domain-filling states with the same symmetry that completely fill the 
domain. The latter are created in primary bifurcations from the conduction state. In the following we describe each of these states in turn. Only steady states are considered.

The domain is periodic in the horizontal with period $6 \lambda_{c}$ in the $x$ and $y$ directions, i.e., the problem is posed on a square lattice. For this reason the $D_{8}$ symmetry cannot be an exact symmetry of any pattern, even if it is well localized.

\section{1. $D_{4}$-symmetric states}

We begin with $D_{4}$-symmetric states. States of this type share the symmetry of the domain and several spatially periodic structures with this symmetry therefore bifurcate from the conduction state. In a $6 \lambda_{c} \times 6 \lambda_{c} \times 1$ domain the first of these states appears at $R a=R a_{c} \approx 66.657$ (Lo Jacono et al 2013) and takes the form of a square pattern oriented along the diagonals, closely followed by a bifurcation to a two-dimensional roll state with rolls oriented along either the $x$ or the $y$ axes. We do not show or discuss these periodic states in this paper. The square state bifurcates subcritically, i.e., towards decreasing $R a$, before turning around to larger values of $R a$ resulting in bistability between the squares and the conduction state. As is well-known from studies of the Swift-Hohenberg model (Burke and Knobloch 2006, Avitabile et al 2010) and related partial differential equations (Lo Jacono et al 2010), secondary bifurcations from subcritical periodic states generate spatially modulated structures, and in large domains these bifurcations take place already at very small amplitude. This is the case here, too, and in the following we focus exclusively on the spatially modulated structures generated through such secondary bifurcations. In the present case these states maintain $D_{4}$ symmetry but become increasingly well localized in space as $R a$ decreases, just as in the Swift-Hohenberg model, before undergoing a complex growth process leading to their interaction with image structures. Figure 1 shows the bifurcation diagram for different types of localized and spatially extended $D_{4}$-symmetric states. The figure shows four solution branches, colored in black, green, light blue and orange. Figure 2 shows examples of typical solutions found along the black, green and light blue branches; these are organized by the state at location (b) indicated in figure 1 and elsewhere by an open red circle.

The green branch extends upwards from this circle and consists of spatially extended but not domain-filling states with $D_{4}$ symmetry, i.e., states that are invariant under the rotations and reflections of a square. In these states convection extends along the diagonals (figure 2, location (d)) resulting in a tesselation of the plane with the periodicity of the domain, i.e., the structures composing the state are connected across the domain boundaries. Because the domain is relatively small and these states respect its symmetry they grow symmetrically as one follows the green branch to larger amplitudes and ultimately succeed in generating a domain-filling state.

Figure 3 describes the green $D_{4}$-symmetric states in more detail. The states take the form of four-armed structures whose arms extend along the diagonals of the domain (figure 3 , location (a)), and the structure within the domain connects with neighboring cells along these diagonals forming the tesselation described above. As one follows the green branch upwards it undergoes a series of back-and-forth oscillations that reflect the nucleation of additional rolls that gradually fill in the voids and generate a domain-filling convective state. This evolution is complete by the time one reaches the prominent left fold in the bifurcation diagram (figure 3, location (d)); with further increase in $R a$ this pattern remains unchanged although it continues to strengthen in amplitude. We mention that the vertical velocity associated with the structures located at the corners of the domain is opposite to that associated with the similar structure at the center of the domain.

The branch below the fold corresponding to the open red circle in figure 1 is colored light blue and also corresponds to $D_{4}$-symmetric states. These states are shown in figure 4 . We see 


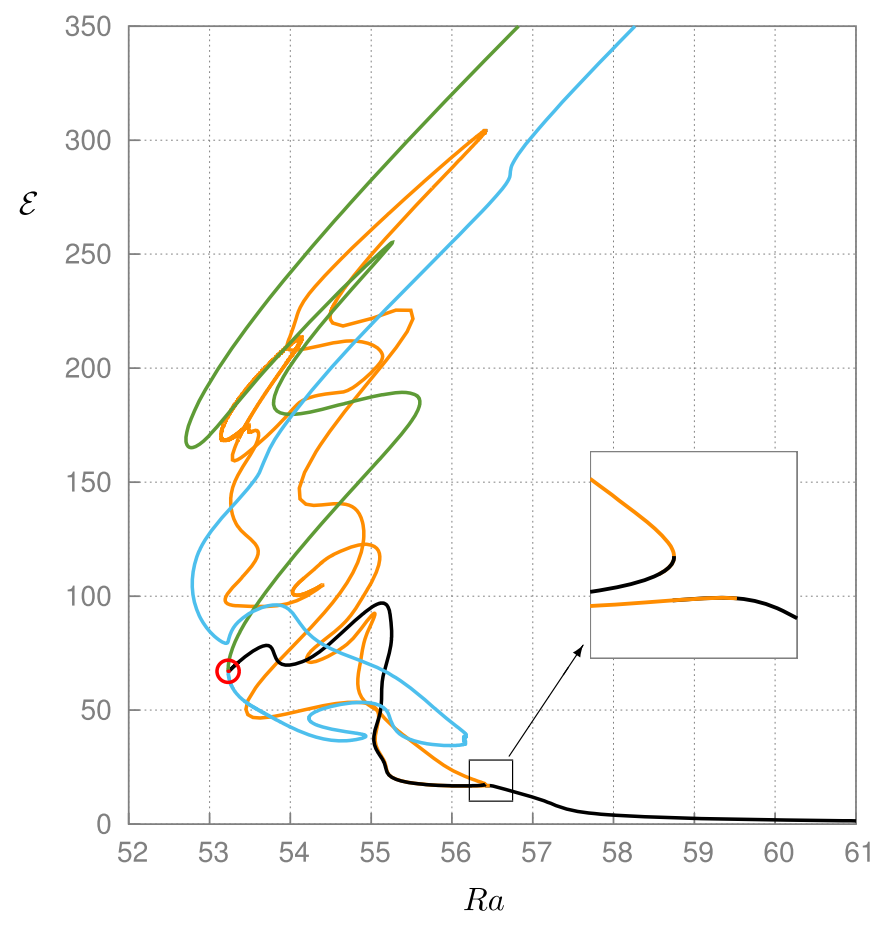

Figure 1. Bifurcation diagram for spatially extended $D_{4}$-symmetric states with four arms along the diagonals (green curve) in a $6 \lambda_{c} \times 6 \lambda_{c} \times 1$ domain showing the kinetic energy $\mathcal{E}$ of the solution as a function of the Rayleigh number $R a$. Superposed is a pseudo-isola of 8-fold states (orange curve) generated via an imperfect bifurcation (see inset) from a branch of localized $D_{4}$-symmetric states (black curve) that originates at small amplitude near $R a_{c} \approx 66.657$ (Lo Jacono et al 2013). These states peak in the center of the domain and differ from the states indicated in light blue which peak at the four corners. The open red circle indicates the bifurcation at which the two types of localized $D_{4}$-symmetric structures (black and light blue curves) terminate on the branch spatially extended $D_{4}$-symmetric states (green curve).

that below this fold the amplitude of the convection in the arms starts to weaken, ultimately leading to a spot-like pattern in which a central spot, characterized by upflow in its center, is surrounded by four spots with downflow in their centers (figure 4, location (d)). As one follows the light blue branch further, it turns towards larger amplitudes. This behavior is a reflection of the onset of a new growth mechanism: instead of evolving extended arms the spot now grows by adding concentric rings. These rings have 8 -fold star-like appearance and lead to the gradual filling in of the voids in the convection pattern (figure 4, location (e) and (f)). Like the green states above the open red circle these new states ultimately also succeed in filling the domain and likewise do so with a $D_{4}$-symmetric pattern (figure 4, location (h), offscale). This pattern is quite different, however, from the $D_{4}$-symmetric domain-filling state at large amplitude along the green branch (figure 3, location (d)). Thus the sequence of transitions along the green/light blue branches can be viewed as a complex transition from one $D_{4}$-symmetric domain-filling state to another. The behavior is reminiscent of a similar complex transition between localized states consisting of rolls parallel to the boundary and a similar state consisting of rolls perpendicular to it, as described by Avitabile et al (2010). 

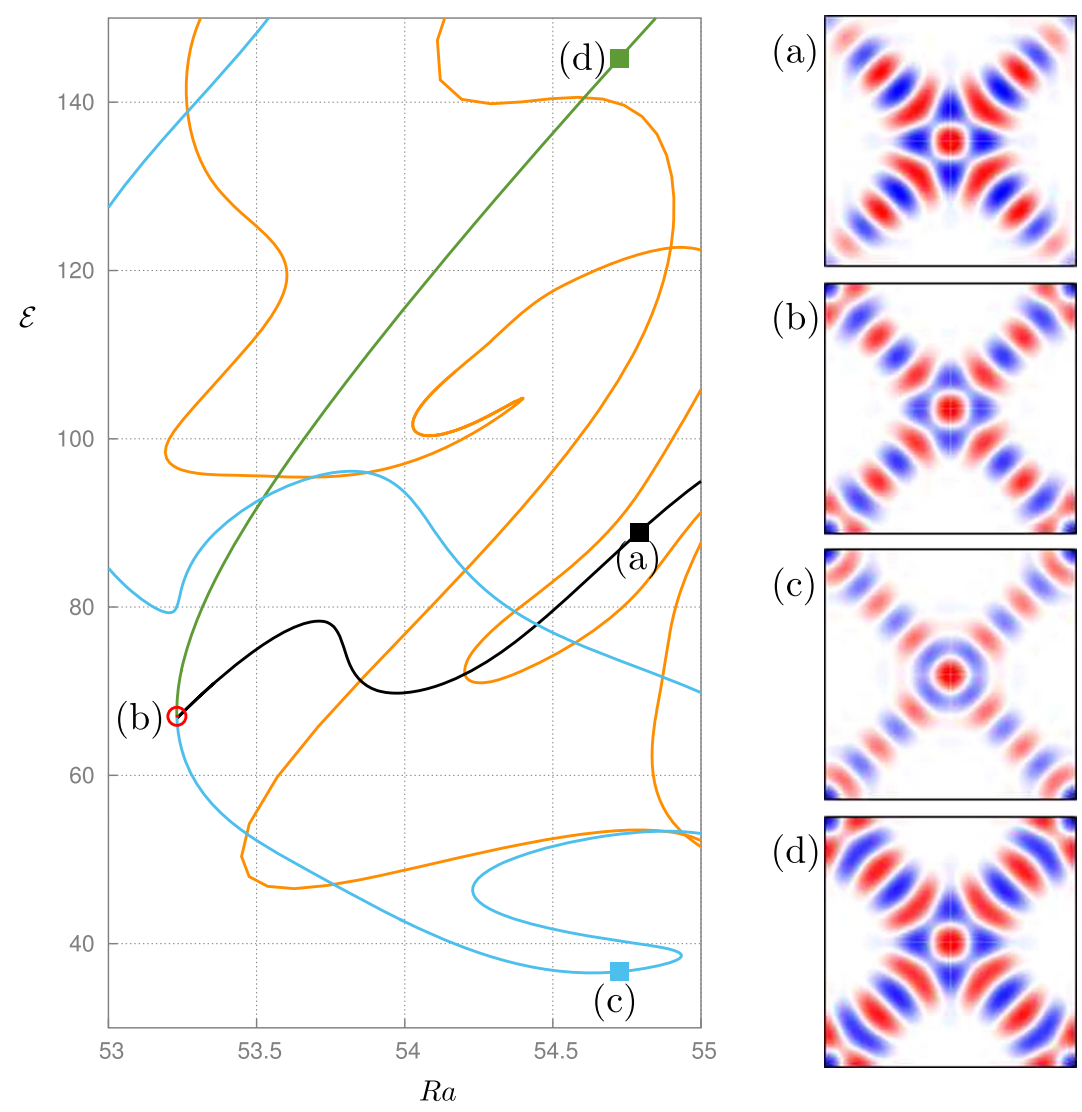

(c)

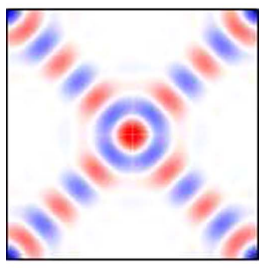

(d)

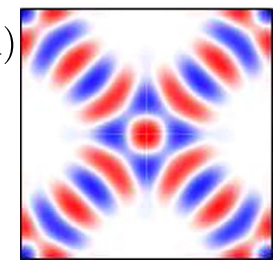

Figure 2. Spatial structures in the vicinity of the open red circle in figure 1, labeled (b), shown in terms of color-coded midlayer vertical velocity (blue: $w(x, y, 1 / 2)<0$, red: $w(x, y, 1 / 2)>0)$. The state (b) interpolates between three distinct state types, labeled (a), (c) and (d). We refer to states of this type as extended, to distinguish them from both localized states and domain-filling states. Domain size: $6 \lambda_{c} \times 6 \lambda_{c} \times 1$.

The secondary bifurcation corresponding to the open red circle in figure 1 provides the key to an additional aspect of the bifurcation diagram. This bifurcation takes place at $R a \approx 53.24$ (figure 2, panel (b), shows the corresponding solution profile) near a fold on the branch of the spatially extended $D_{4}$-symmetric states, and also leads to spatial modulation (black curve), but this time the holes in the pattern are located at the corners of the domain (figure 5, location (d)) in contrast to the behavior shown in figure 4. This bifurcation, like that leading to the appearance of holes in figure 4 , is entirely analogous to the bifurcation terminating the snaking branches of localized states in the one-dimensional Swift-Hohenberg model (Burke and Knobloch 2006) in the sense that it is the result of an Eckhaus instability of a periodic pattern (Bergeon et al 2008). There too this bifurcation creates holes in an otherwise periodic state, and just as in the Swift-Hohenberg model, increasing $R a$ leads to deeper holes and ultimately the breaking of the connections between adjacent structures at the corners of the domain. Thus the bifurcation indicated by the open red circle represents the termination of a branch of $D_{4}$-symmetric spatially localized structures on a branch of $D_{4}$-symmetric but spatially extended structures. 


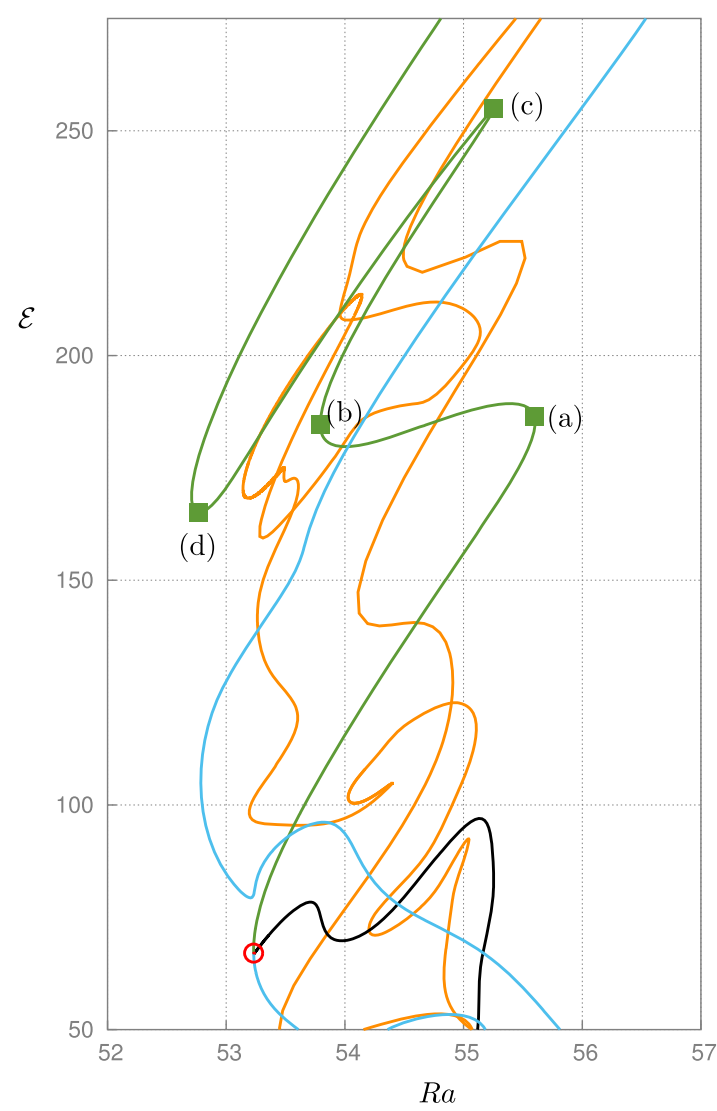

(a)

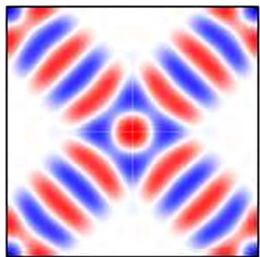

(b)

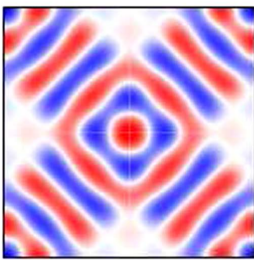

(c)

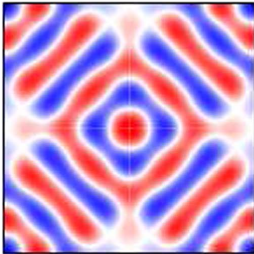

(d)

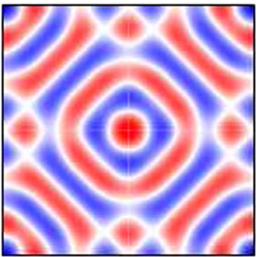

Figure 3. Spatial structure along the branch of spatially extended four-armed $D_{4}$-symmetric states with arms along the diagonals (green curve) at (a) a right fold at $R a \approx 55.60$, (b) the next left fold at $R a \approx 53.79$, (c) the next right fold at $R a \approx 55.25$, and (d) the final left fold at $R a \approx 52.77$, showing the growth of the structure and its approach to a domain-filling convective state. The solution profiles are shown in terms of color-coded midlayer vertical velocity (blue: $w(x, y, 1 / 2)<0$, red: $w(x, y, 1 / 2)>0)$. A key state, indicated by an open red circle here and in figure 1 , is shown separately in figure 2 (b). Domain size: $6 \lambda_{c} \times 6 \lambda_{c} \times 1$.

Complications arise, however, as this branch is continued farther away from the secondary bifurcation. Initially, the pattern evolves as expected from the above analogy with the one-dimensional Swift-Hohenberg equation (figure 5, locations (c) and (d)), before collapsing into an axisymmetric target pattern (figure 5, location (b)). Because this state is localized it is almost insensitive to the boundaries of the domain. However, just before (b) the branch undergoes a fold (figure 1, inset) and starts to grow again, forming once again an 8-fold target pattern with identical fingers that grow out along both the principal axes of the domain and its diagonals (figure 5, location (a)). We indicate the resulting branch in orange (figure 5). Of course the apparent $D_{8}$ symmetry of this state cannot be exact—-the distant $D_{4}$-boundaries must break the $D_{8}$ symmetry at some level, albeit very weakly while the state remains well localized.

In figure 6 we show a succession of states further up this branch. At location (a) the state has developed into an eight-armed state with arms that extend along both the axes and the 


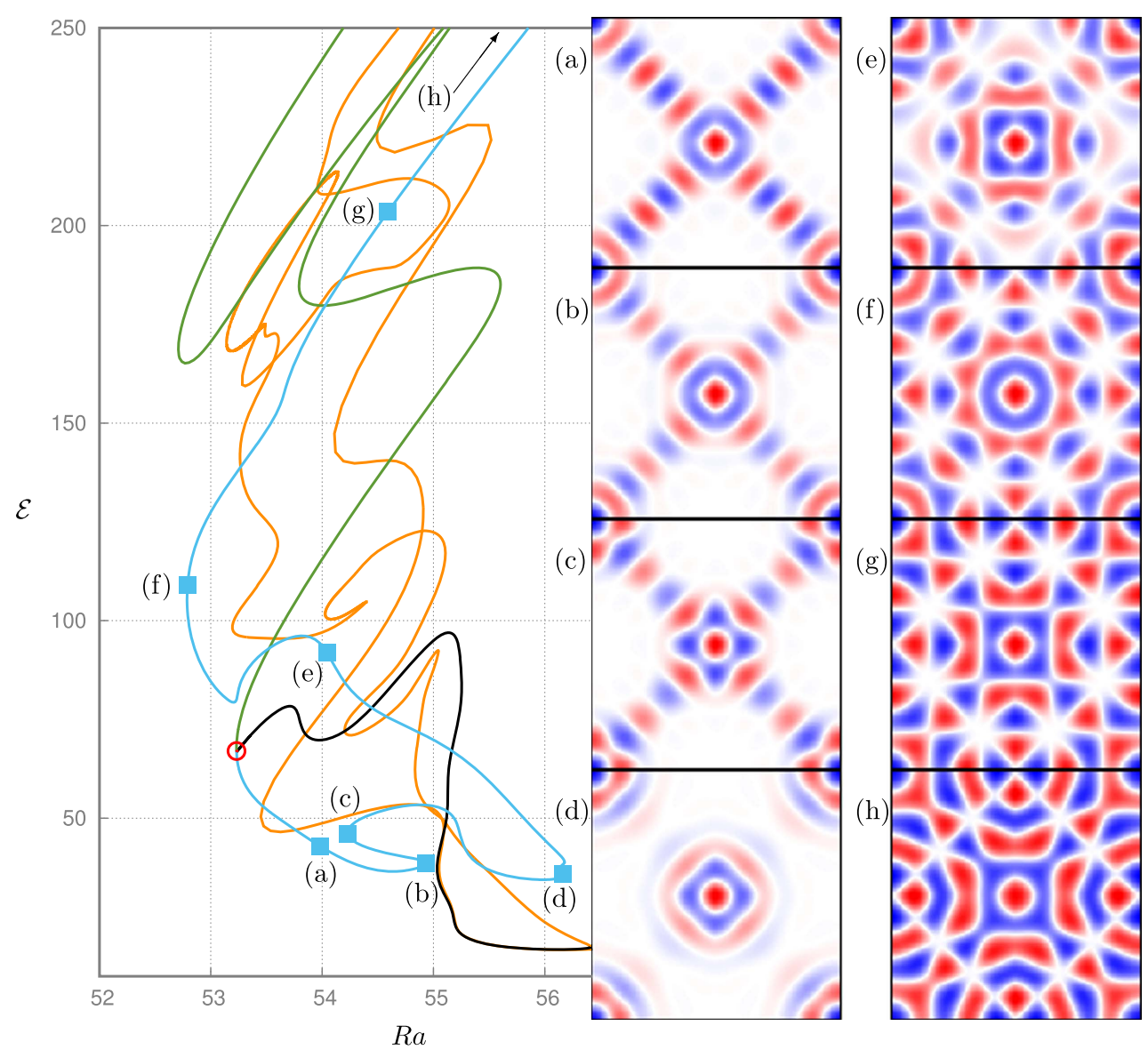

Figure 4. Spatial structure along the light blue branch of spatially extended four-armed $D_{4}$-symmetric states below the open red circle in figure 1 showing a gradual transition from a four-armed state to a $D_{4}$-symmetric domain-filling state via a spot-like pattern at location (d). The final state, shown at location (h), differs from the $D_{4}$-symmetric domain-filling state in figure 3 , location (d), obtained by following the green branch above the open red circle. The solution profiles are shown in terms of color-coded midlayer vertical velocity (blue: $w(x, y, 1 / 2)<0$, red: $w(x, y, 1 / 2)>0)$. Domain size: $6 \lambda_{c} \times 6 \lambda_{c} \times 1$.

diagonals of the domain. However, because of the longer arms along the diagonals the $D_{8}$ symmetry is quite strongly broken and the arms along the diagonals now differ from those along the principal axes. With further increase in amplitude the arms located along the diagonals shrink but those along the axes grow in length until the structure makes contact with image structures located in adjacent cells (figure 6, location (b)). After this first contact the diagonal arms begin to lengthen again and the structure makes contact with image structures along the diagonals as well (figure 6, location (d)). As one follows the branch to yet larger amplitude the voids in the pattern start to fill in but a domain-filling structure is not reached. Instead the branch turns around towards smaller amplitude, and gradually loses the arms along the principal axes, leaving only arms along the diagonals. At these locations the structures closely resemble those shown in figure 5 at locations (d) and ultimately (c) and (b), 


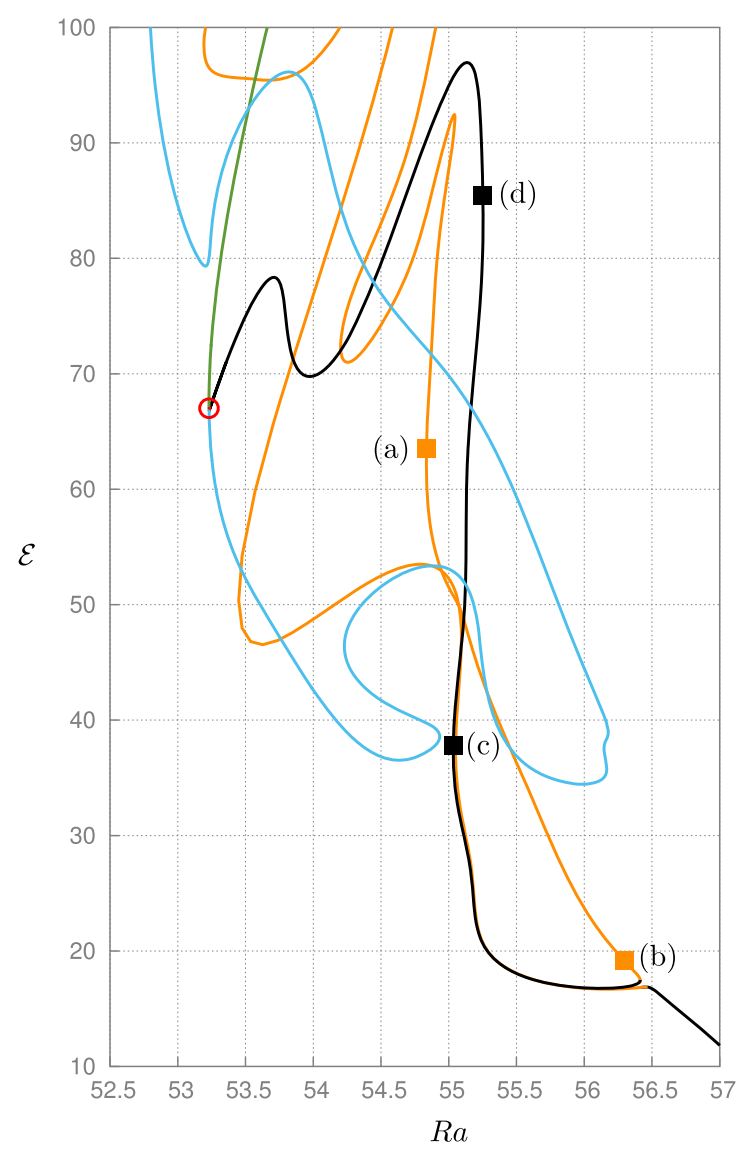

(a)

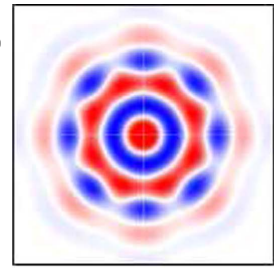

(b)

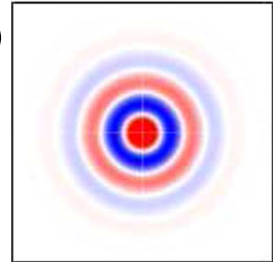

(c)

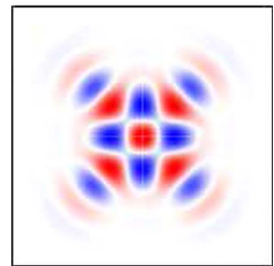

(d)

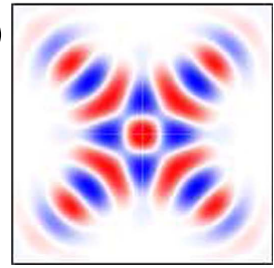

Figure 5. The transition from a four-armed structure with arms oriented along the diagonals (panel $(\mathrm{c}), R a \approx 55.03$, and panel (d), $R a \approx 55.25$, black curve) to an eightarmed structure with arms oriented along both the diagonals and the principal axes of the domain (panel (a), $R a \approx 54.84$, orange curve) via a target pattern (panel (b), $R a \approx 56.29)$. The solution profiles are shown in terms of color-coded midlayer vertical velocity (blue: $w(x, y, 1 / 2)<0$, red: $w(x, y, 1 / 2)>0)$. Domain size: $6 \lambda_{c} \times 6 \lambda_{c} \times 1$.

i.e., the branch returns to the vicinity of location (b) where the solution takes the form of an axisymmetric target pattern. Thus the branch of the approximately $D_{8}$-symmetric star-like patterns forms a large pseudo-isola. Were it not for the effect of the domain boundaries the imperfect bifurcation visible below location (b) and the associated minute splitting of the two branches emanating towards smaller $R a$ would be absent and the target pattern would likely be found at the location of the bifurcation from the $D_{4}$-symmetric localized states to $D_{8}$-symmetric localized structures, mediating the transition between states (a) and (c) in figure 6 . We surmise that the complex behavior located near $R a \approx 55, \mathcal{E} \approx 52$ where the light blue branch comes very close to the slightly split black/orange branches corresponds to the location of the other end of the branch of the $D_{8}$-symmetric states, where the $D_{4}$-symmetric states start to develop arms along the principal axes of the domain. Once again this connection is apparently broken by the presence of $D_{4}$-symmetric boundaries not too far away from the localized 8-fold structure. We conclude that the presence of the complex pseudo-isola of 


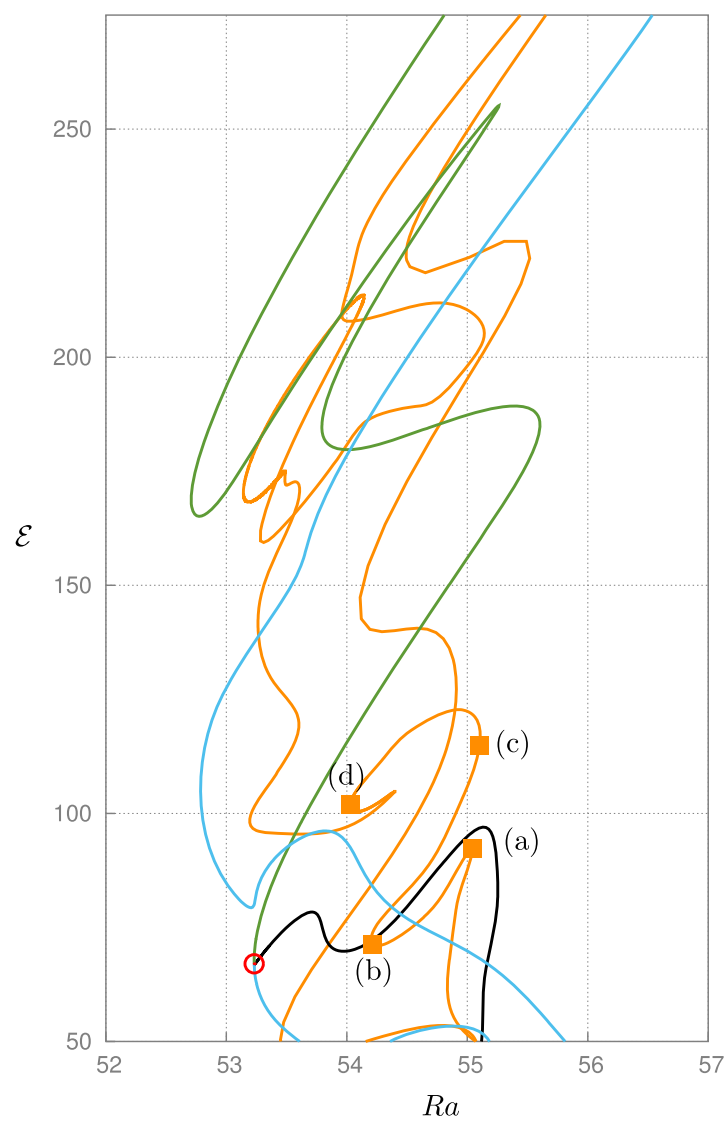

(a)

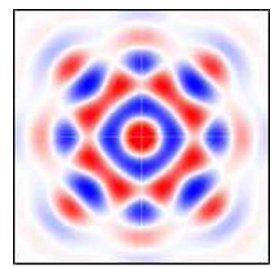

(b)

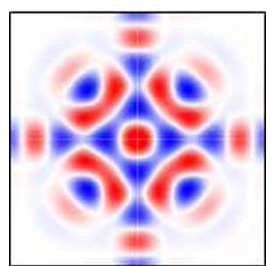

(c)

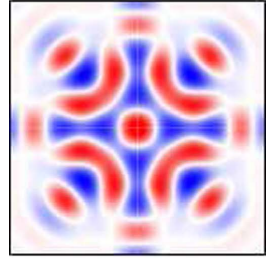

(d)

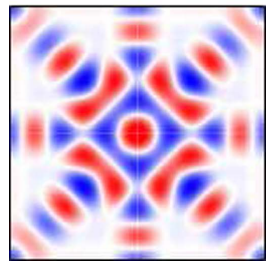

Figure 6. Continuation of figure 5 showing the evolution of the solutions along the pseudo-isola (orange curve). Spatial structure of an 8 -fold state at (a) a right fold at $R a \approx 55.05$, (b) the next left fold at $R a \approx 54.22$, (c) the next right fold at $R a \approx 55.10$ and $(\mathrm{d})$ the next left fold at $R a \approx 54.03$, showing the growth of an eight-armed structure as one proceeds upwards along the orange branch. The solution profiles are shown in terms of color-coded midlayer vertical velocity (blue: $w(x, y, 1 / 2)<0$, red: $w(x, y, 1 / 2)>0)$. Domain size: $6 \lambda_{c} \times 6 \lambda_{c} \times 1$.

8 -fold states is a consequence of the finite periodicity of the domain and in particular its $D_{4}$ symmetry which is incompatible with 8 -fold structures as soon as these reach the size of the domain.

Because of the presence of an imperfect bifurcation below location (b) we restore the black color to the branch that extends from (b) to smaller amplitudes. This branch can be continued all the way to its origin in a secondary bifurcation at very small amplitude from a subcritical domain-filling state that bifurcates from the conduction state in a primary bifurcation at $R a=R a_{c} \approx 66.657$.

\section{2. $D_{2}$-symmetric states}

We now turn to states for which a reflection in the diagonal acts by -1 , i.e., $D_{2}$-symmetric states. Because of their different symmetry these states lie on a distinct solution branch but are 


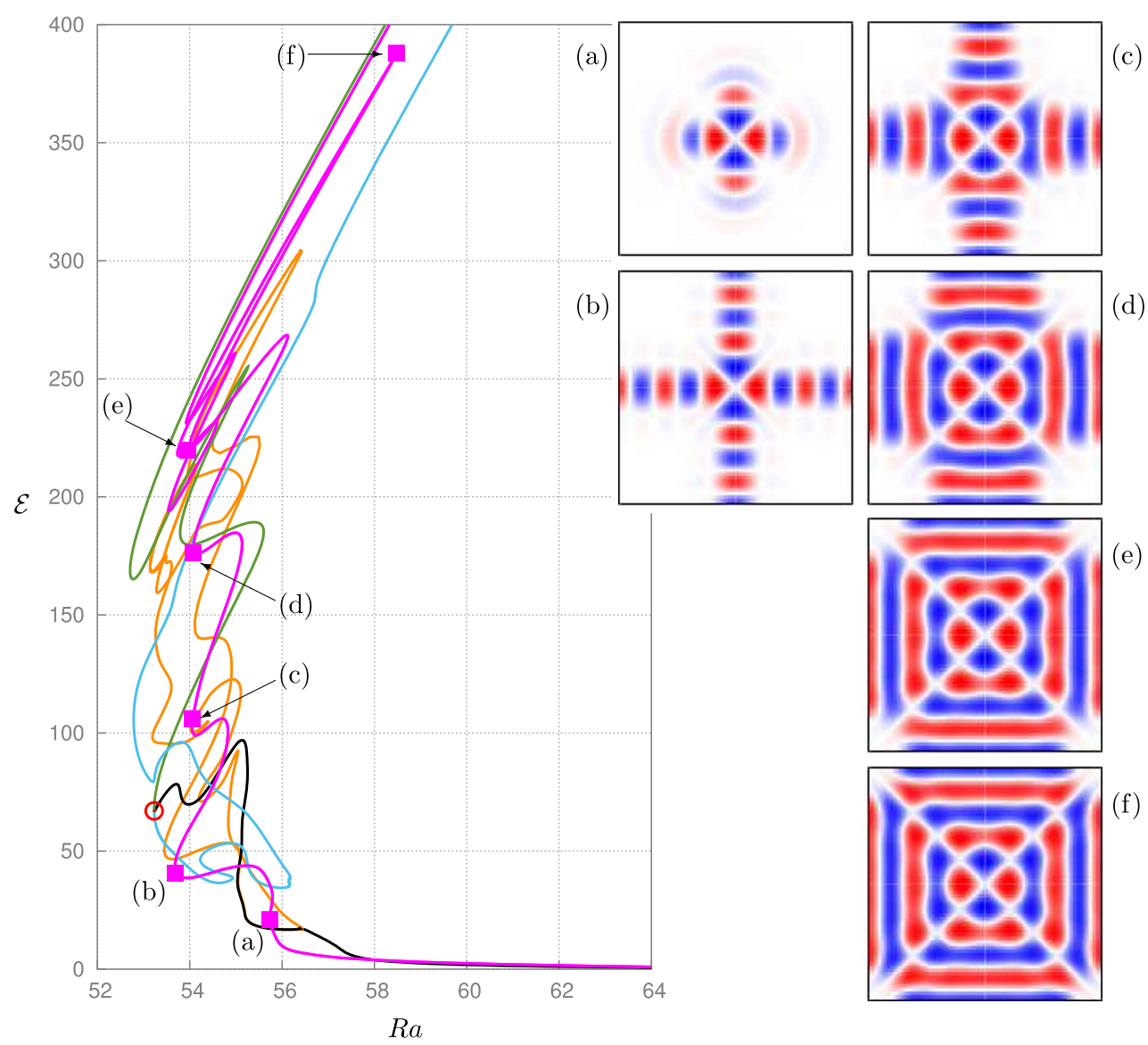

Figure 7. Spatial structure of $D_{2}$-symmetric states with four arms oriented along the principal axes at several different locations along the (pink) solution branch. The first five of these locations correspond to the first five left folds ((a) $R a \approx 55.73$, (b) $R a \approx 53.69$, (c) $R a \approx 54.05$, (d) $R a \approx 54.07$, (e) $R a \approx 53.96$ ); the last panel (f) shows an almost domain-filling state at $R a \approx 58.48$. The solution profiles are shown in terms of color-coded midlayer vertical velocity (blue: $w(x, y, 1 / 2)<0$, red: $w(x, y, 1 / 2)>0)$. The branches of $D_{4}$-symmetric states from figure 1 are included in the left panel for comparison. Domain size: $6 \lambda_{c} \times 6 \lambda_{c} \times 1$.

also characterized by four arms. However, this time the arms are oriented along the principal axes of the domain. Figure 7 shows the resulting solution branch and the evolution of these states towards a domain-filling state. In each profile the $D_{2}$ symmetry is most easily discerned by comparing the structure at its center with that present in the $D_{4}$-symmetric states shown in figures $3-5$.

It is noteworthy that despite the complexity of the states just described an (unstable) small amplitude target pattern always manages to grow into a domain-filling state, as one traces out the solution branch, by nucleating additional convection rolls in appropriate locations. As we shall see next this is apparently no longer the case in larger domains. 


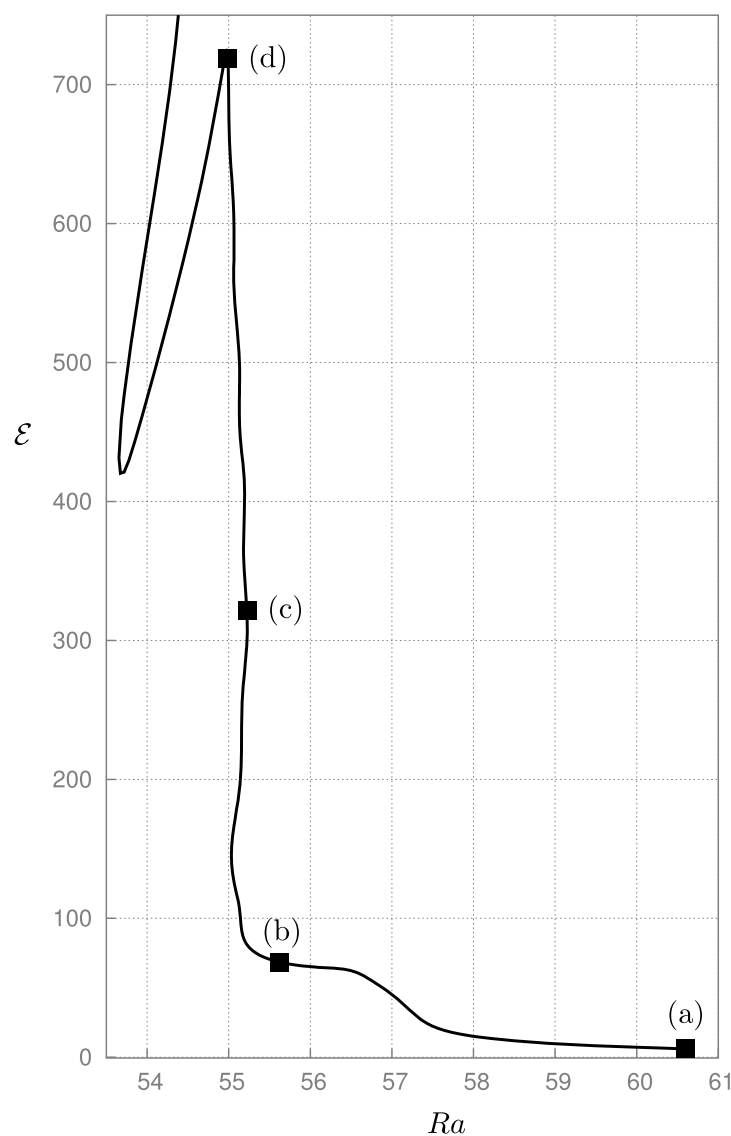

(a)

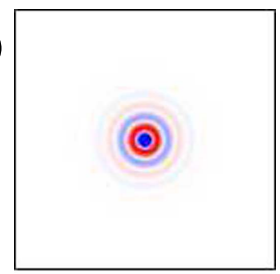

(b)

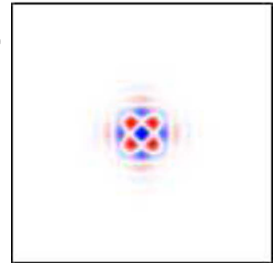

(c)

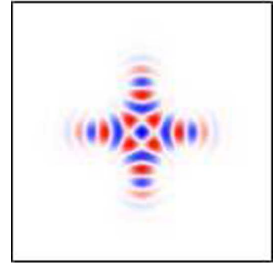

(d)

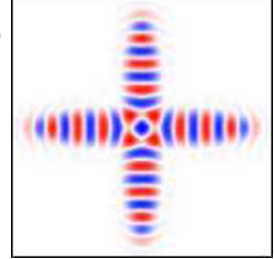

Figure 8. Left panel: bifurcation diagram showing the kinetic energy $\mathcal{E}$ of $D_{4}$-symmetric solutions as a function of the Rayleigh number $R a$ in a $12 \lambda_{c} \times 12 \lambda_{c} \times 1$ domain. Right panels: a small amplitude target pattern at location (a) $R a \approx 60.60$ and $D_{4}$-symmetric localized states at location (b) $R a \approx 55.62$, (c) $R a \approx 55.22$ and (d) $R a \approx 54.99$, all shown in terms of color-coded midlayer vertical velocity (blue: $w(x, y, 1 / 2)<0$, red: $w(x, y, 1 / 2)>0)$. The corresponding locations are indicated in the left panel. Domain size: $12 \lambda_{c} \times 12 \lambda_{c} \times 1$.

\section{Results: $12 \lambda_{c} \times 12 \lambda_{c} \times 1$ domain}

As in the $6 \lambda_{c} \times 6 \lambda_{c} \times 1$ domain we first perform a direct numerical simulation of the governing equations to identify an unstable target pattern and use this to initialize numerical continuation. As shown in figure 8 when the Rayleigh number is decreased from $R a=60.60$ the target state develops into a $D_{4}$-symmetric localized structure by initially nucleating cells along each diagonal (figure 8(b)); additional protocells are visible along the principal axes and these start developing into finger-like structures at the first saddle-node along the branch. Figure 8(c) shows the resulting structure at the second saddle-node. Each of the fingers resembles the worm state present in the Swift-Hohenberg equation (Avitabile et al 2010), i.e., an elongated segmented structure, spatially localized in two dimensions, consisting of transverse rolls (Joets and Ribotta 1988, Dennin et al 1996, Lo Jacono et al 2013). 


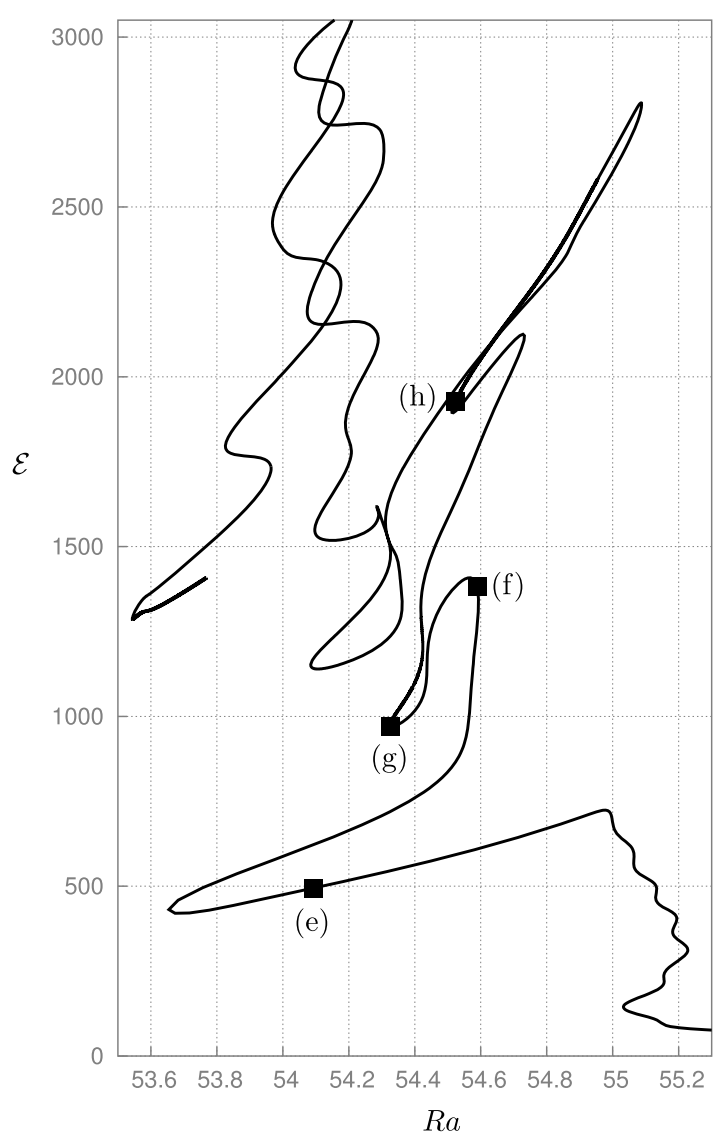

(e)

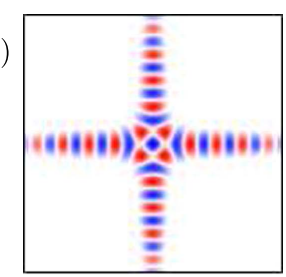

(f)

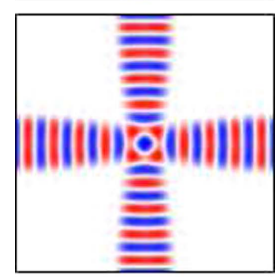

(g)

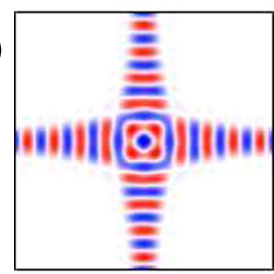

(h)

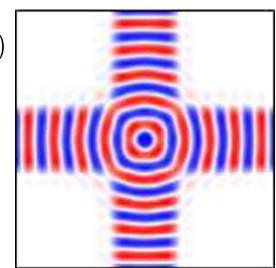

Figure 9. Left panel: continuation of the bifurcation diagram in figure 8. Right panels: cross-like structures at locations (e) $R a \approx 54.09$, (f) $R a \approx 54.59$, (g) $R a \approx 54.33$, (h) $R a \approx 54.52$ indicated in the left panel, showing the growth of the ring structure around the core. The branch has been terminated at an arbitrary location near $R a \approx 53.8$. Domain size: $12 \lambda_{c} \times 12 \lambda_{c} \times 1$.

As one proceeds to higher amplitude following the complex gyrations of the $D_{4}$ solution branch (figure 9) the arms grow in length by nucleating new rolls at their tips until they start to interact with their images across the domain boundary. This growth process is reflected in the snaking of the solution branch most clearly visible in the lower right corner of the left panel in figure 9. At the same time the width of the arms is a sensitive function of the Rayleigh number $R a$ and hence oscillates rapidly as the branch zigzags across the snaking region. The interaction with image structures first occurs near $R a=55$ and thereafter the number of rolls in each arm remains constant and equal to ten. It is this interaction that is responsible for the abrupt change in slope ('corner') in the bifurcation diagram (figure 8(d)). As one continues to follow the branch, the lower values of $R a$ lead to a decrease in its amplitude owing to progressive thinning of the arms (figure 9(e)). Above the leftmost saddlenode, at $R a=53.6$, the arms start to thicken and the amplitude starts to increase again until the next saddle-node on the right where the branch turns to the left again (figure 9(f)). Beyond this saddle-node four roll segments, one from each worm and adjacent to the core structure, connect and form a new ring around the center of the localized structure; the ring becomes 


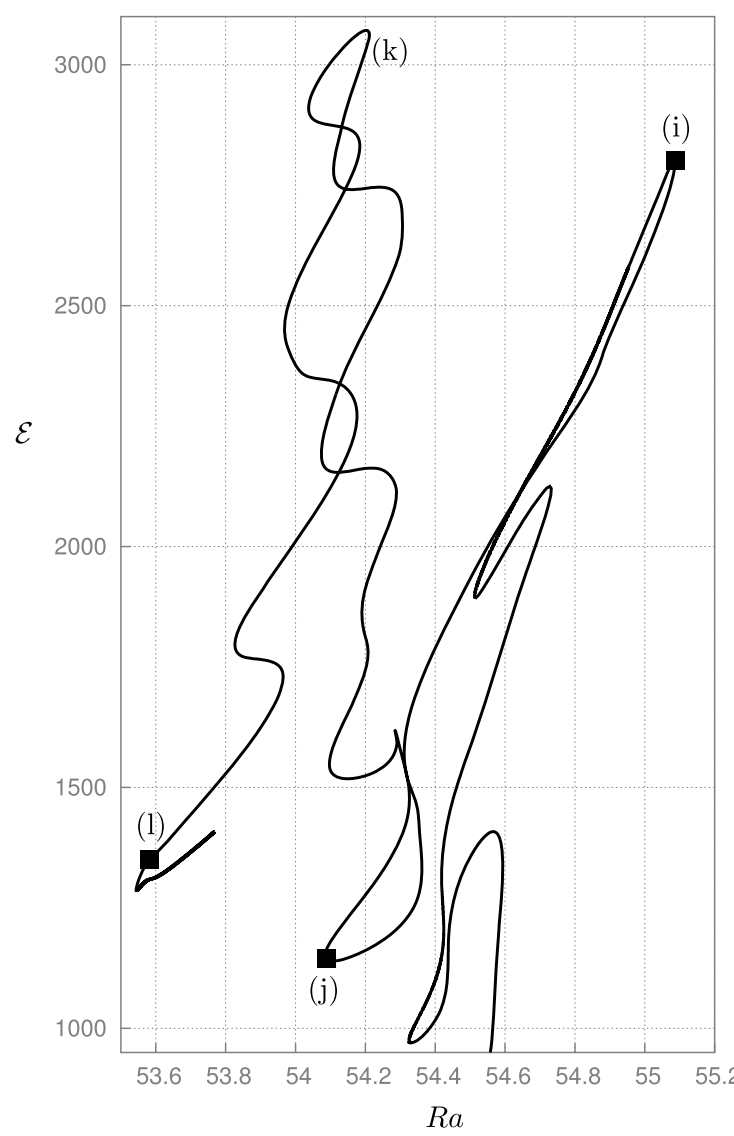

(i)

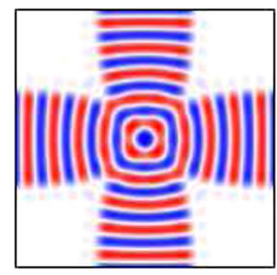

(j)

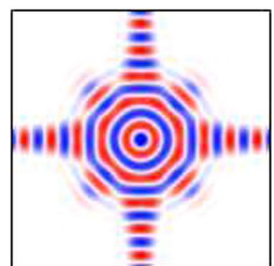

(k)

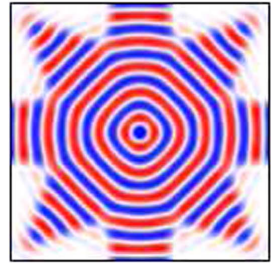

(1)

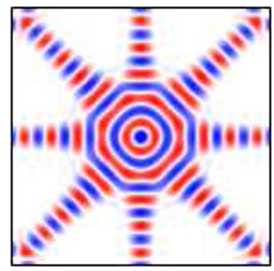

Figure 10. Left panel: detail of the bifurcation diagram in figure 9. Right panels: cross-like structures at locations (i) $R a \approx 55.087$, (j) $R a \approx 54.088$, showing the transition from cross-like growth to star-like growth, and fully developed starfish structures at locations (k) $R a \approx 54.189$, (1) $R a \approx 53.582$, all indidated in the left panel. The branch has been terminated at an arbitrary location near $R a \approx 53.8$. Domain size: $12 \lambda_{c} \times 12 \lambda_{c} \times 1$.

fully developed by the next saddle-node on the left (figure $9(\mathrm{~g})$ ). The next set of structures on the right is associated with the formation of the next complete ring around the core (figure 9(h)) culminating in a Celtic cross-like structure at the rightmost saddle-node at $R a=55.14$ (figure 10(i)). Thus up to this point the structure has grown primarily in a radially symmetric fashion. Beyond this point the growth of the structure changes qualitatively, and the structure sends out fingers along the diagonals (figure 10(j)). These fingers grow until they reach the corners of the domain (figure 10(k)) a fact that is responsible for the termination of the tower-like feature in the bifurcation diagram at $\mathcal{E} \approx 3000$. Thereafter the structure fills in the corners, resembling an eight-armed starfish at $R a=53.57$ (figure 10(1)). We believe that the growth of the structure continues beyond the state shown in figure 10(1) and that ultimately the structure fills all of the available two-dimensional domain, likely forming a twodimensional labyrinthine pattern with $D_{4}$ symmetry.

We have not computed $D_{2}$-symmetric state for this domain size. 


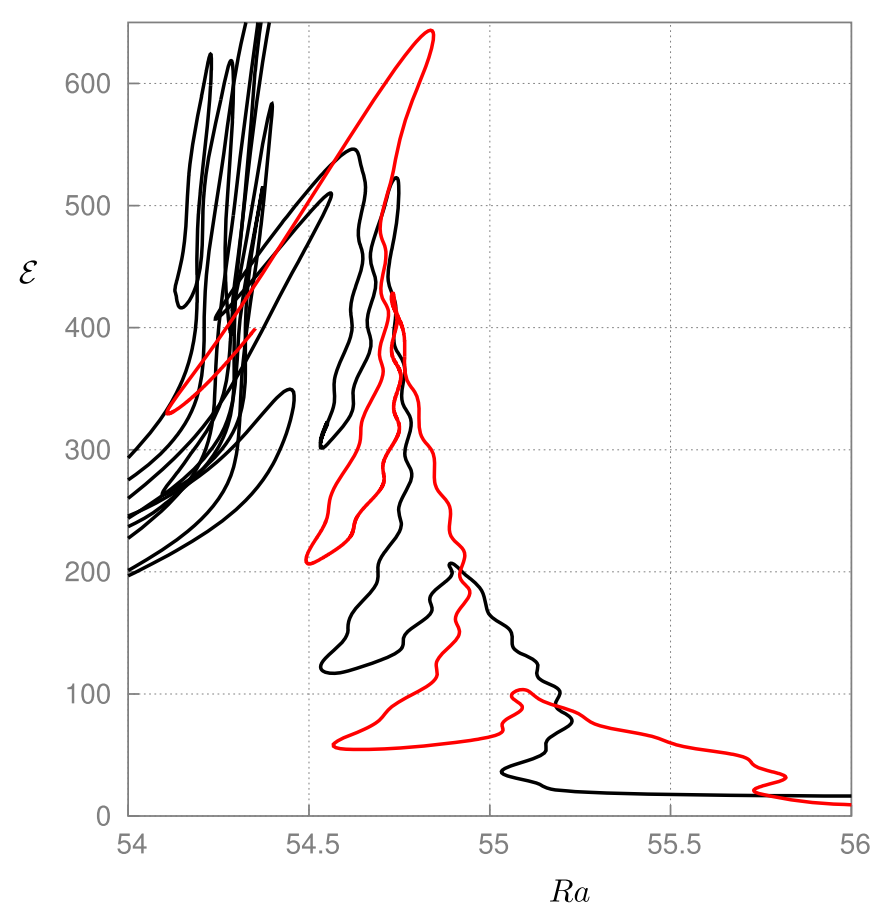

Figure 11. Bifurcation diagram for $D_{4}$-symmetric states (black curve) and $D_{2}$-symmetric states (red curve). The small oscillations in the center of the diagram correspond to nucleation of new rolls at the tips of the arms, while the large back-and-forth excursions are associated with the addition of a complete ring around the core. See Lo Jacono et al (2013) for details. This behavior terminates in prominent folds at the top center of the diagram, heralding the onset of interaction with image structures (top left of diagram). Domain size: $18 \lambda_{c} \times 18 \lambda_{c} \times 1$.

\section{Results: $18 \lambda_{c} \times 18 \lambda_{c} \times 1$ domain}

Fully spatially localized structures in an $18 \lambda_{c} \times 18 \lambda_{c} \times 1$ domain were presented in an earlier paper (Lo Jacono et al 2013). Here we extend these results by following the branches of $D_{4}$ - and $D_{2}$-symmetric states into regimes where the localized structures begin to interact with their images. Figure 11 summarizes what we know in terms of a bifurcation diagram with the black curve representing $D_{4}$-symmetric states and the red curve $D_{2}$-symmetric states.

\section{1. $D_{4}$-symmetric states}

We begin by describing the behavior of the $D_{4}$-symmetric states computed in Lo Jacono et al (2013) once these start to interact with their images. Figure 12(a) shows a $D_{4}$-symmetric state with four arms centered on the origin just prior to their length reaching half the domain size, i.e., prior to the first interaction with image states. This state corresponds to the last prominent fold on the right of the bifurcation diagram in the left panel beyond which the ordered growth described in Lo Jacono et al (2013) ceases. Figure 12(b) shows a state at a right fold on one of the horizontally extended loops that form the subsequent tangle, while figure 12(c) shows the corresponding state near the next left fold. Finally, figure 12(d) shows the state at the next 


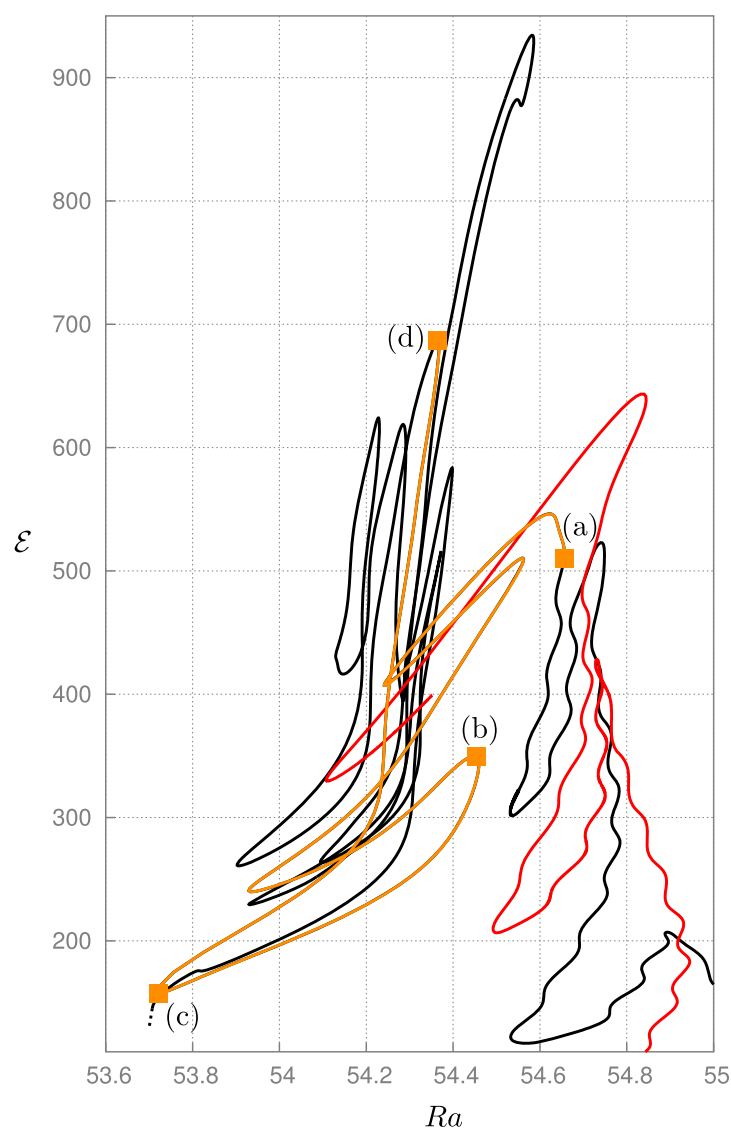

(a)

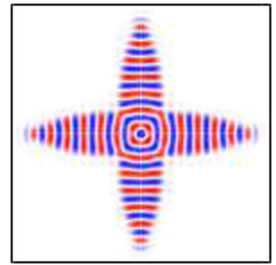

(b)

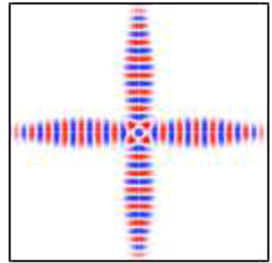

(c)

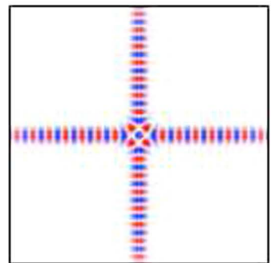

(d)

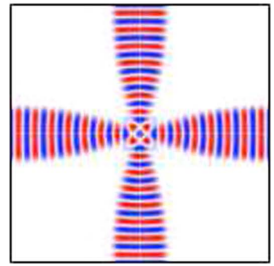

Figure 12. Left panel: detail of the bifurcation diagram in figure 11, with the black curve representing $D_{4}$-symmetric states and the red curve $D_{2}$-symmetric states. Right panels: four-armed $D_{4}$-symmetric states at locations (a) $R a \approx 54.66$, (b) $R a \approx 54.37$, (c) $R a \approx 54.28$ and (d) $R a \approx 54.36$ along the orange portion of the branch shown in the left panel, representing the continuation of the branch of $D_{4}$-symmetric localized states (black curve), all shown in terms of color-coded midlayer vertical velocity (blue: $w(x, y, 1 / 2)<0$, red: $w(x, y, 1 / 2)>0)$. Domain size: $18 \lambda_{c} \times 18 \lambda_{c} \times 1$.

right, upward tilted fold, showing that each arm is now bulging out at the location of contact with image arms, in contrast, for example, to figure 12(b).

Figure 13 shows the evolution further along the solution branch and reveals that the prominent upwards extended loop corresponds to notable broadening of the arms of the structure. Note in particular the changes that take place in the core between panel (f) and (g) resulting in the formation of a hole in the core of the structure.

As in the previous case the solution branch does not terminate and appears to continue forever. We base this understanding on the fact that the wavenumber along the horizontally extended branches is quantized owing to the spatial periodicity in this direction. As a result, once the arms touch the number of horizontal wavelengths is necessarily fixed, here equal to 16. Hence the only degree of freedom permitted to the (horizontal) arms is their width. As a result when a branch enters a particular region of parameter space the different states at this location will in general differ in their widths, and since this is a continuous variable we 


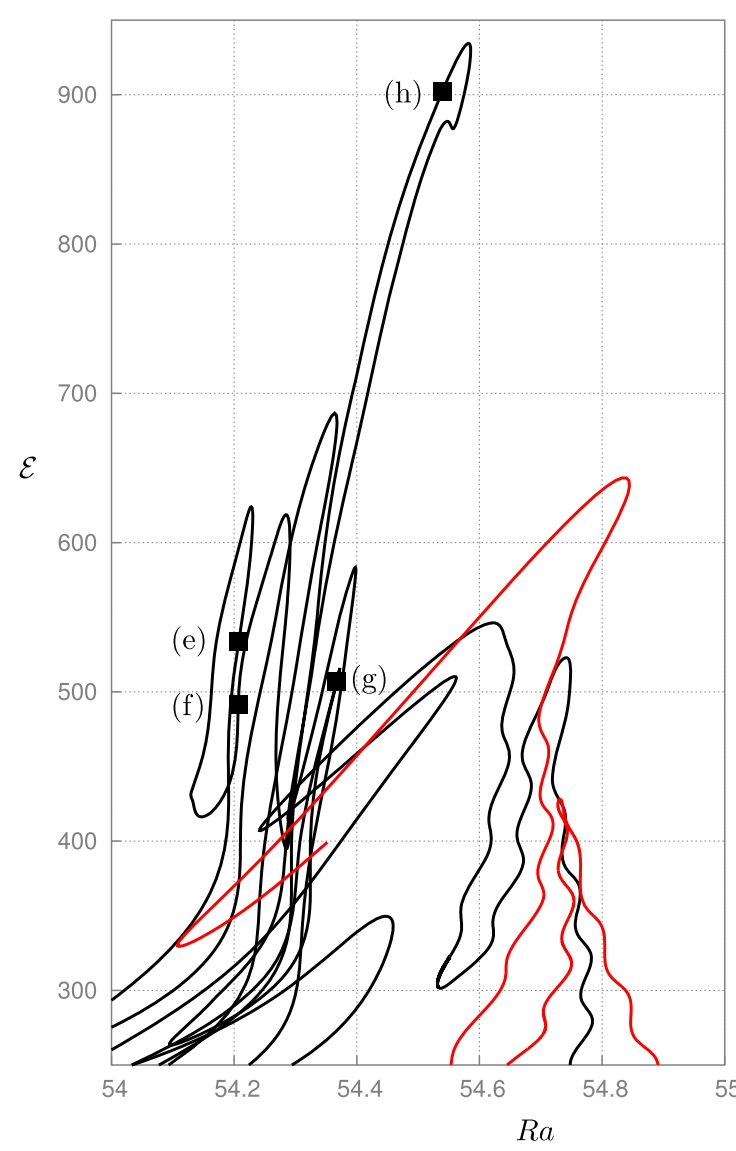

(e)

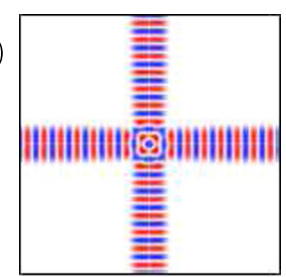

(f)

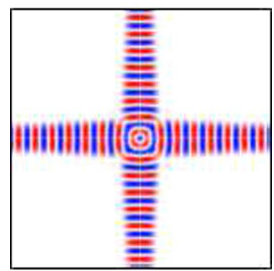

(g)

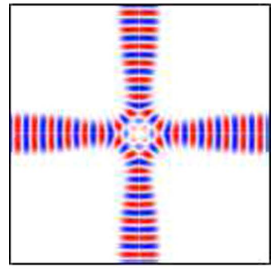

(h)

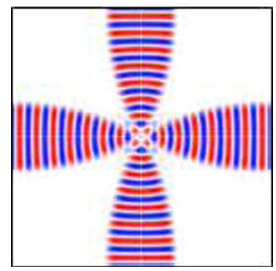

Figure 13. Left panel: detail of the bifurcation diagram in figure 11, with the black curve representing $D_{4}$-symmetric states and the red curve $D_{2}$-symmetric states. Right panels: four-armed $D_{4}$-symmetric state at locations (e) $R a \approx 54.207$, (f) $R a \approx 54.206$, (g) $R a \approx 54.366$ and $(\mathrm{h}) R a \approx 54.539$ shown in the left panel, all shown in terms of color-coded midlayer vertical velocity (blue: $w(x, y, 1 / 2)<0$, red: $w(x, y, 1 / 2)>0$ ). Domain size: $18 \lambda_{c} \times 18 \lambda_{c} \times 1$.

conjecture that the branch can oscillate forever without closing or ending, potentially generating a space-filling curve.

We have not continued this branch further but note that we have detected no evidence that the solution may ultimately generate additional arms and so attempt to approach a state of convection that fills the whole domain. We surmise that instead the branch will undergo steeper and steeper vertical excursions corresponding to rapid growth in width: abrupt growth in the branch corresponds to abrupt increase in width, with no snaking, that takes place when the pair of fronts bounding the arms laterally on either side see a translation-invariant roll structure inbetween, see Avitabile et al (2010). Of course, when this happens the rolls that extend from the horizontal arms in the vertical direction will collide with those extending horizontally from the vertical arms leading to a state that fills the domain but for a line of defects along the four diagonals. 


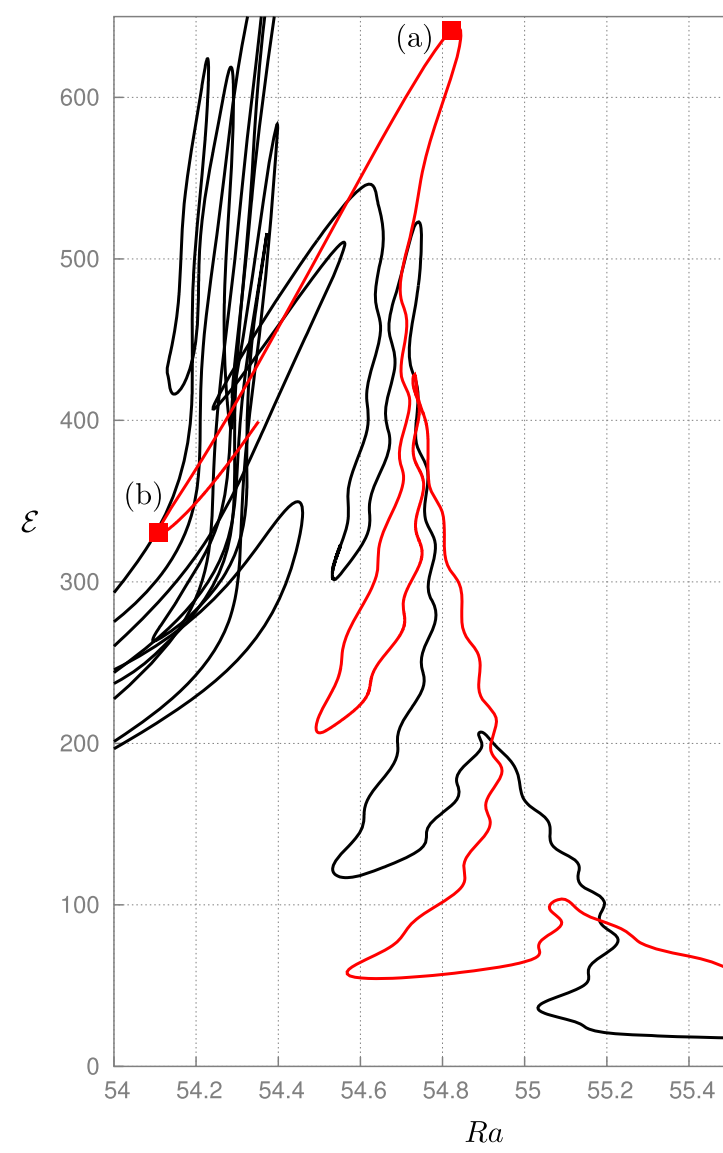

(a)

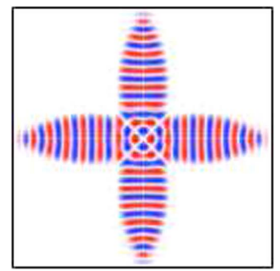

(b)

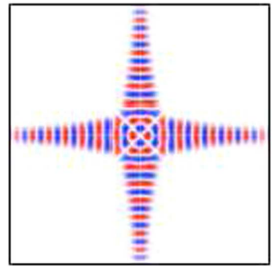

Figure 14. Left panel: detail of the bifurcation diagram in figure 11, with the black curve representing $D_{4}$-symmetric states and the red curve $D_{2}$-symmetric states. Right panels: four-armed $D_{2}$-symmetric states at locations (a) $R a \approx 54.82$, (b) $R a \approx 54.11$ shown in the left panel, both shown in terms of color-coded midlayer vertical velocity (blue: $w(x, y, 1 / 2)<0$, red: $w(x, y, 1 / 2)>0)$. Domain size: $18 \lambda_{c} \times 18 \lambda_{c} \times 1$.

\section{2. $D_{2}$-symmetric states}

The growth of spatially localized $D_{2}$-symmetric states is documented in Lo Jacono et al (2013). As the resulting solution branch (red curve in figure 11) is followed the four arms lengthen and begin to interact with their images. As in the $D_{4}$-symmetric case this interaction is associated with the prominent fold at the top right of the diagram. Figure 14(a) shows the solution in the vicinity of this fold while figure 14(b) shows the corresponding solution at the next left fold. Although we have not followed this branch further we anticipate that the evolution of the solutions along the branch will recapitulate that found for the $D_{4}$-symmetric states subject to reflections in the diagonals acting by -1 as opposed to +1 in the $D_{4}$-symmetric case.

\section{Discussion}

From the above pictures we abstract two basic principles. The localized structure grows continuously in area, with qualitative changes in the bifurcation diagram associated with 
interactions with periodically replicated images. However, the amplitude of the solution, here measured by the total kinetic energy $\mathcal{E}$, does not increase monotonically. This is because the arms of the structure shrink transversely as the Rayleigh number decreases and swell as it increases. Indeed, in the $12 \lambda_{c} \times 12 \lambda_{c} \times 1$ domain we find that for $R a \lesssim 54.6$ the arms of the cross thin with distance from the core but thicken with distance for $R a \gtrsim 54.6$, with $R a \approx 54.6$ playing the role of a Maxwell point at which the energy along the arms of the structure is independent of the distance from the core, with like behavior observed in other domains. Similar behavior is observed in individual worms as described by the SwiftHohenberg equation SH35 (Avitabile et al 2010),

$$
u_{t}=-\mu u-\left(\nabla^{2}+1\right)^{2} u+b_{3} u^{3}-u^{5},
$$

where $b_{3}$ is a positive constant that determines the extent of bistability between the trivial state $u=0$ representing the conduction state and a periodic state $u(x, y)$, here a roll pattern, representing convection. The symmetry $u \rightarrow-u$ of equation (7) plays the role of the midplane reflection symmetry of equations (1)-(4) when the boundary conditions (6) are used. Equation (7) exhibits worm-like states that are fully localized in two spatial dimensions; these worms snake when continued in $\mu$, at least initially, where $\mu$ is to be identified with $R a_{c}-R a$. Moreover, multiple disconnected branches of worms are present (Avitabile et al 2010). These reveal a strong selection between skinny worms at the right of the snaking region (larger $\mu$ ) and pudgy worms at the left of the snaking region (smaller $\mu$ ). Additional rolls start to nucleate in the middle of the associated snaking region and do so simultaneously at both tips (head and tail) of the worm. The increased radius of curvature of the worm demands a larger wavenumber along the periphery and this change of wavenumber is enough to pin the front between the roll state and the homogeneous state and maintain a steady state.

\section{Conclusion}

In this paper we have computed spatially localized states with $D_{4}$ and $D_{2}$ symmetry in threedimensional binary fluid convection in a porous medium with three different aspect ratios and identified a series of remarkable convective structures in this relatively simple system. The solutions are born at small amplitude through the gradual transformation of a small amplitude target pattern. The details of the resulting growth process depend sensitively on the aspect ratio of the domain, with the structures in the smallest domain growing much as expected on the basis of existing studies of the one-dimensional bistable Swift-Hohenberg equation (7). In particular the structure ultimately approaches a domain-filling convection pattern except for the residual presence of defects along the diagonals or in the four corners. However, in larger domains it appears that the system finds it increasingly difficult to grow into a domain-filling state and we have been unable to follow the solution branches into a regime beyond cross-like structures that interact with their periodically distributed nearest neighbors.

The initial phase of growth from an axisymmetric spot or target patterns takes the form of $D_{4}$-symmetric growing dendrites and resembles the fingering of moving fronts in $\mathrm{SH} 35$ resulting from a depinning instability as described in the Swift-Hohenberg context by Burke and Knobloch (2007). The growth during this phase is well-ordered and takes the form of nucleation of new rolls at the tips of the arms interspersed with reconnection events resulting in the growth of the core of the structure. In the small domain (section 2 ) the $D_{4}$-symmetric states take the form of structures elongated along the diagonals while the $D_{2}$-symmetric states take the form of a cross. In the intermediate domain (section 3) $D_{4}$-symmetric states are of cross type and continue to be so in the largest domain examined. In this domain (section 4) 
the $D_{2}$-symmetric states are also of cross type. Once the arms have grown to an extent that they interact with image states the structure of the bifurcation diagrams becomes immensely complex and reflects some of the phenomena associated with the interaction of one-dimensional localized states with the domain boundary investigated in detail by Bergeon et al (2008) and via matched asymptotic expansions near a codimension two point by Kozyreff et al (2009). The resulting patterns resemble convex and concave structures often seen in liquids with surface tension except that here the structures appear to be confined by the concentration field whose gradients are expelled from regions of active convection (Lo Jacono et al 2013). This mechanism is, we believe, ultimately behind the profusion of solution branches characteristic of this problem, of which we have reported on only the simplest.

A question that arises inevitably concerns the stability of the states we have uncovered. In earlier study of this same system on an $18 \lambda_{c} \times 18 \lambda_{c} \times 1$ domain (Lo Jacono et al 2013) we have performed a series of direct numerical simulations with imposed $D_{4}$ or $D_{2}$ symmetry and showed that subject to this constraint the portions of the branches of $D_{4}$ and $D_{2}$-symmetric states lying above left folds were indeed stable as expected on the basis of standard homoclinic snaking theory (Knobloch 2015). We believe that these conclusions carry over to the smaller domains studied here. In particular, we expect that the almost domain-filling states we have found in the $6 \lambda_{c} \times 6 \lambda_{c} \times 1$ domain are stable.

\section{Acknowledgments}

The initial phase of this project was supported by the National Science Foundation under grant DMS-0908102; the work was completed with support from grant DMS-1613132.

\section{References}

Alonso A, Mercader I and Batiste O 2014 Fluid Dyn. Res. 46041418

Assemat P, Bergeon A and Knobloch E 2007 Phys. Fluids 19104101

Avitabile D, Lloyd D J B, Burke J, Knobloch E and Sandstede B 2010 SIAM J. Appl. Dyn. Syst. 9704

Batiste O and Knobloch E 2005 Phys. Rev. Lett. 95244501

Batiste O, Knobloch E, Alonso A and Mercader I 2006 J. Fluid Mech. 560149

Beaume C, Bergeon A and Knobloch E 2013a Phys. Fluids 25024105

Beaume C, Knobloch E and Bergeon A 2013b Phys. Fluids 25114102

Bergeon A, Burke J, Knobloch E and Mercader I 2008 Phys. Rev. E 78046201

Bergeon A and Knobloch E 2008a Physica D 2371139

Bergeon A and Knobloch E 2008b Phys. Fluids 20034102

Blanchflower S 1999 Phys. Lett. A 26174

Blanchflower S and Weiss N 2002 Phys. Lett. A 294297

Burke J and Knobloch E 2006 Phys. Rev. E 73056211

Burke J and Knobloch E 2007 Chaos 17037102

Coullet P, Riera C and Tresser C 2000 Phys. Rev. Lett. 843069

Dawes J H P 2007 J. Fluid Mech. 570385

Dennin M, Ahlers G and Cannell D S 1996 Phys. Rev. Lett. 772475

Duguet Y, Schlatter P and Henningson D S 2009 Phys. Fluids 21111701

Ghorayeb K 1997 Etude des écoulements de convection thermosolutale en cavité rectangulaire Thèse de Doctorat l'Université Paul Sabatier, Toulouse 3, France

Ghorayeb K and Mojtabi A 1997 Phys. Fluids 92339

Golubitsky M, Swift J W and Knobloch E 1984 Physica D 10249

Joets A and Ribotta R 1988 Phys. Rev. Lett. 602164

Knobloch E 2015 Annu. Rev. Condens. Matter Phys. 6325

Kozyreff G, Assemat P and Chapman S J 2009 Phys. Rev. Lett. 103164501 
Lloyd D J B and Sandstede B 2009 Nonlinearity 22485

Lloyd D J B, Sandstede B, Avitabile D and Champneys A R 2008 SIAM J. Appl. Dyn. Syst. 71049

Lo Jacono D, Bergeon A and Knobloch E 2010 Phys. Fluids 22073601

Lo Jacono D, Bergeon A and Knobloch E 2013 J. Fluid Mech. 730 R2

McSloy J M, Firth W J, Harkness G K and Oppo G-L 2002 Phys. Rev. E 66046606

Mercader I, Alonso A and Batiste O 2008 Phys. Rev. E 77036313

Schneider T M, Gibson J F and Burke J 2010a Phys. Rev. Lett. 104104501

Schneider T M, Marinc D and Eckhardt B 2010b J. Fluid Mech. 646441

Silber M and Knobloch E 1988 Phys. Rev. A 381468

Umla R, Augustin M, Huke B and Lücke M 2010 J. Fluid Mech. 649165 